\title{
Intracoronary Hydatid Cyst Resulted in Coronary Artery Disease in a Young Patient
}

\author{
Unsal Vural' ${ }^{1}, M D$; Ahmet Arif Aglar ${ }^{1}$, MD; ilyas Kayacioglu' ${ }^{1}, M D$
}

DOI: $10.21470 / 1678-9741-2018-0033$

\begin{abstract}
Among all cystic echinococcosis cases, only $0.5 \%-2 \%$ exhibit a cardiac involvement. Only $10 \%$ of these become symptomatic. Considering the long time interval between the start of infestation and symptoms to occur, it is hard to diagnose cystic echinococcosis. When detected, even if it is asymptomatic, intramyocardial hydatid cyst requires surgical intervention due to risks of spontaneous rupture and anaphylaxis. In literature, no case of hydatid cyst located in the coronary arterial wall has been reported. Twenty-two-year-old male patient with previous history of pulmonary cystic echinococcosis was referred to us with typical symptoms of coronary artery disease. Coronary cineangiography revealed proximal left diagonal artery (LAD) occlusion. Pre-operative transthoracic echocardiography of the
\end{abstract}

patient planned to undergo coronary artery bypass grafting unveiled an intracoronary calcified cystic mass. In operation, the calcified cystic mass with well-defined borders and size of $2 \times 2 \mathrm{~cm}$ located within wall of proximal segment of the LAD artery was excised and double bypass with left internal thoracic artery (LITA) and great saphenous vein grafts to the LAD and first diagonal arteries, respectively, was done. Pathological analysis of the mass revealed it to be an inactive calcified hydatid cyst. Echinococcal IgG-ELISA test was positive. 12-week oral albendazole treatment (2x400 mg/day) was launched postoperatively and the patient was discharged on $7^{\text {th }}$ postoperative day.

Keywords: Albendazole. Parasitology. Echinococcosis. Heart Disease.

\begin{tabular}{ll}
\hline \multicolumn{2}{l}{ Abbreviations, acronyms \& symbols } \\
\hline CT & = Computed tomography \\
ECG & $=$ Echocardiography \\
LAD & $=$ Left diagonal artery \\
LITA & $=$ Left internal thoracic artery \\
\hline
\end{tabular}

\section{INTRODUCTION}

The liver, followed by the lung, is the most common site of involvement for the hydatid cyst. The incidence of concomitant liver and lung hydatidosis varies from 5.8 to $13.3 \%{ }^{[1]}$. These cysts grow at a rate of roughly $1 \mathrm{~cm}$ per year, and become symptomatic within 5 to 10 years ${ }^{[2]}$. Parasitic larvae migrate to heart mainly via coronary circulation. Frequency of cardiac hydatidosis vary from 0.5 to $2 \%{ }^{[3]}$. The embryo reaches full growth at 1 to 5 years after being lodged in the heart. The myocardial reaction to the cyst creates an adventitial pericyst layer ${ }^{[4]}$. We aimed to present a

'Departament Cardiovascular Surgery, Doktor Siyami Ersek Gogus Kalp ve Damar Cerrahisi Egitim ve Arastirma Hastanesi Ringgold Standard Institution, Istanbul, Turkey.

This study was carried out at the Department of Cardiovascular Surgery, Doktor Siyami Ersek Gogus Kalp ve Damar Cerrahisi Egitim ve Arastirma Hastanesi Ringgold Standard Institution, Istanbul, Turkey.

No financial support.

No conflict of interest rarely-seen location and thus complication, namely the coronary artery disease, of cardiac hydatidosis to attention.

\section{CASE REPORT}

A 22-year old male patient was referred to our clinic with exertional angina. His history revealed pulmonary hydatidosis treated through cystectomy and capitonnage followed by 12week oral albendazole treatment (400 mg/twice a day) five years ago. Chest $X$-ray and thoracic computed tomography (CT) exposed only few scattered calcifications within pulmonary parenchyma (Figure 1). Cineangiography of the patient with ST segment depression in exercise test revealed that the left diagonal artery (LAD) and $1^{\text {st }}$ diagonal arteries were proximally occluded (Figure 2). In transthoracic echocardiography, a cystic mass of $2 \times 2 \mathrm{~cm}$ with well-defined borders was detected on the left ventricular anterior wall (Figure 3). The patient was taken into operation for coronary artery bypass grafting. Operation was carried out under cardiopulmonary bypass instituted after median sternotomy. The mass, with size of $2 \times 2 \mathrm{~cm}$ and regular

Correspondence Address:

Unsal Vural

(iD) http://orcid.org/0000-0002-2338-8541

Doktor Siyami Ersek Gogus Kalp ve Damar Cerrahisi Egitim ve Arastirma Hastanesi

Cardiovascular surgery

Tıbbiye Cad. Uskudar Istannbul Istanbul 34688, Turkey

E-mail: unsalvural@gmail.com 


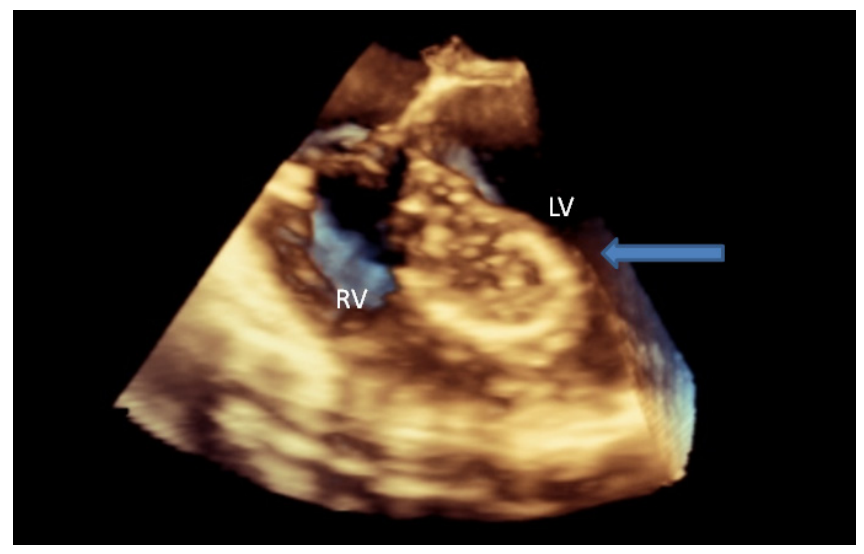

Fig. 1 - Echocardiographic view of the hydatid cyst. $L V=l e f t$ ventricle; $R V=$ right ventricle

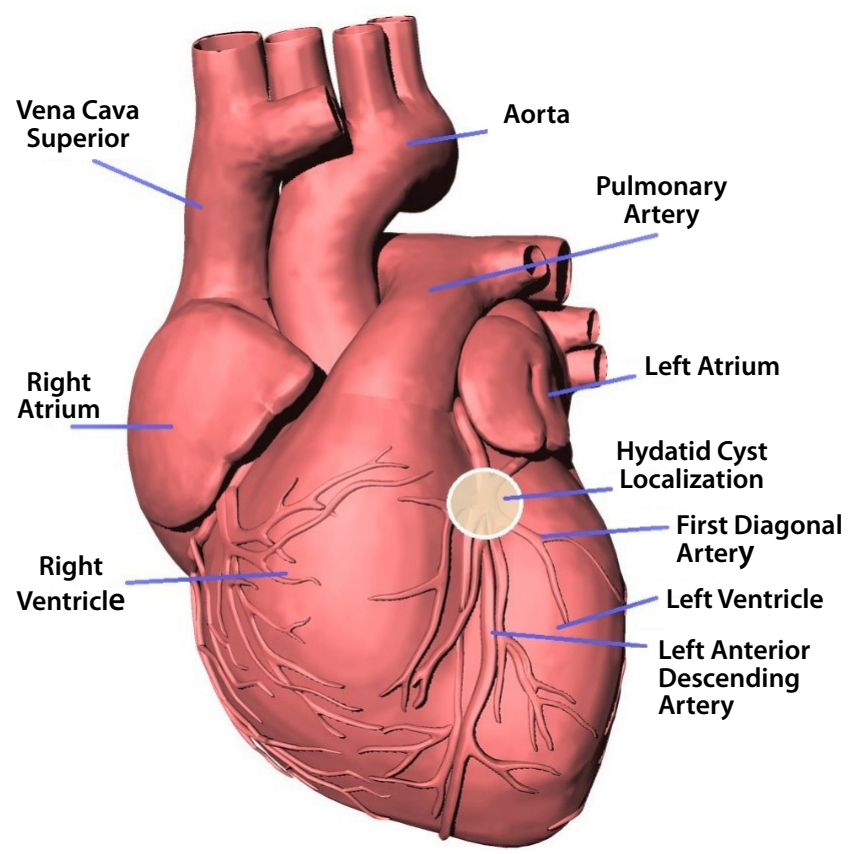

Fig. 2 - Localization of the intracoronary hydatid cyst.

borders, was found to be located between the left main and LAD coronary arteries (Figure 3). Since calcified, the cyst was isolated en bloc with the coronary artery segments it had infiltrated (Figure 3). Free ends of the coronary arteries opening into the cavity left behind after cyst excision were ligated. Capitonnage was performed after irrigation with hypertonic saline solution. Following that, LAD artery and $1^{\text {st }}$ diagonal artery were bypassed. Macroscopically, it was detected that the cyst contains clear colorless fluid (eau de rock). Microbiological and pathological analysis of both the cyst and its ingredients revealed findings consistent with the hydatid cyst. Echinococcal IgG-ELISA test was found to be positive (sensitivity: $94 \%$, specificity: $99 \%)^{[5]}$. The patient received oral albendazole treatment $(10 \mathrm{mg} / \mathrm{kg} /$ day; $2 \times 400 \mathrm{mg} /$ day) postoperatively for 12 weeks. During 1-year follow-up, diagnostic tests were negative and no recurrence was observed.

\section{DISCUSSION}

Cardiac hydatidosis involves mainly the left ventricle (55-60\%) due to its dominant circulatory network, followed, in decreasing incidence, by right ventricle (15\%), interventricular septum (9\%), left atrium (8\%), pericardium (7\%), pulmonary artery (6-7\%), right atrium (4\%), and interatrial septum (2\%) ${ }^{[6]}$. However, to date, no coronary arterial involvement of hydatid cyst causing coronary artery occlusion has been reported.

Though coughing, palpitation and angina could be the symptoms, only $10 \%$ of the cardiac hydatidosis cases become symptomatic ${ }^{[7]}$. In symptomatic cases, since potential cyst rupture could end up with anaphylaxis, early surgical intervention is recommended ${ }^{[8]}$. Ben-Hamda et al. ${ }^{[8]}$ reported that hydatidosis, particularly in elderly patients, could mimic angina and myocardial infarction as a result of compression to adjacent tissues. In younger patients, symptoms may comprise rather of dyspnea, coughing, loss of weight, and fever ${ }^{[9]}$. Though the case we present is young, he presented symptoms typical of elders. Rupture into cardiac chambers may cause ventricular outflow tract obstruction, low cardiac output syndrome, pulmonary, cerebral or peripheral emboli and hypertension. In case of conduction system involvement, rhythm problems can occur $^{[8]}$. Moreover, as in our case, referral with coronary artery disease related symptoms is possible, too. Q and inverted T waves seen in ECG can be helpful in diagnosis. Echocardiography is an effective tool in diagnosis of cardiac hydatidosis. CT and magnetic resonance imaging are effective, particularly in diagnosis of extracardiac hydatidosis. In the case we present, intracoronary calcified inactive cyst is thought to be originated from embolization of ruptured pulmonary hydatid cyst since his history reveals previous pulmonary involvement. It is postulated that, as a result of either previous medical treatment or spontaneous cyst inactivation, the cystic growth was impeded, and the cyst was calcified. Therefore, the cyst was easily enucleated. When findings suggestive of cardiac involvement are present, particularly in endemic areas (Mediterranean coasts, Middle East, Australia, South America), cardiac hydatidosis must be considered ${ }^{[2]}$.

Surgical intervention is the first-line treatment since medical therapy is insufficient in preventing the rupture of the cyst ${ }^{[8]}$. As in the case we present, coronary complication of pulmonary hydatidosis could be seen even 5 years after the treatment. Some surgeons proposed perioperative sterilization of the cystic location with either $2 \%$ formalin or $20 \%$ hypertonic saline solution ${ }^{[2]}$. The cyst in our case was easily isolated en bloc with coronary artery segments it infiltrated without any rupture. Free ends of the coronary arteries opening into the cavity left behind after cyst excision were ligated. Capitonnage was performed after irrigation with hypertonic saline solution. Following that, LAD artery and $1^{\text {st }}$ diagonal artery were bypassed.

Definitive diagnosis and assessment of cystic activity are done through cyst serology. Though echinococcal IgG-ELISA 

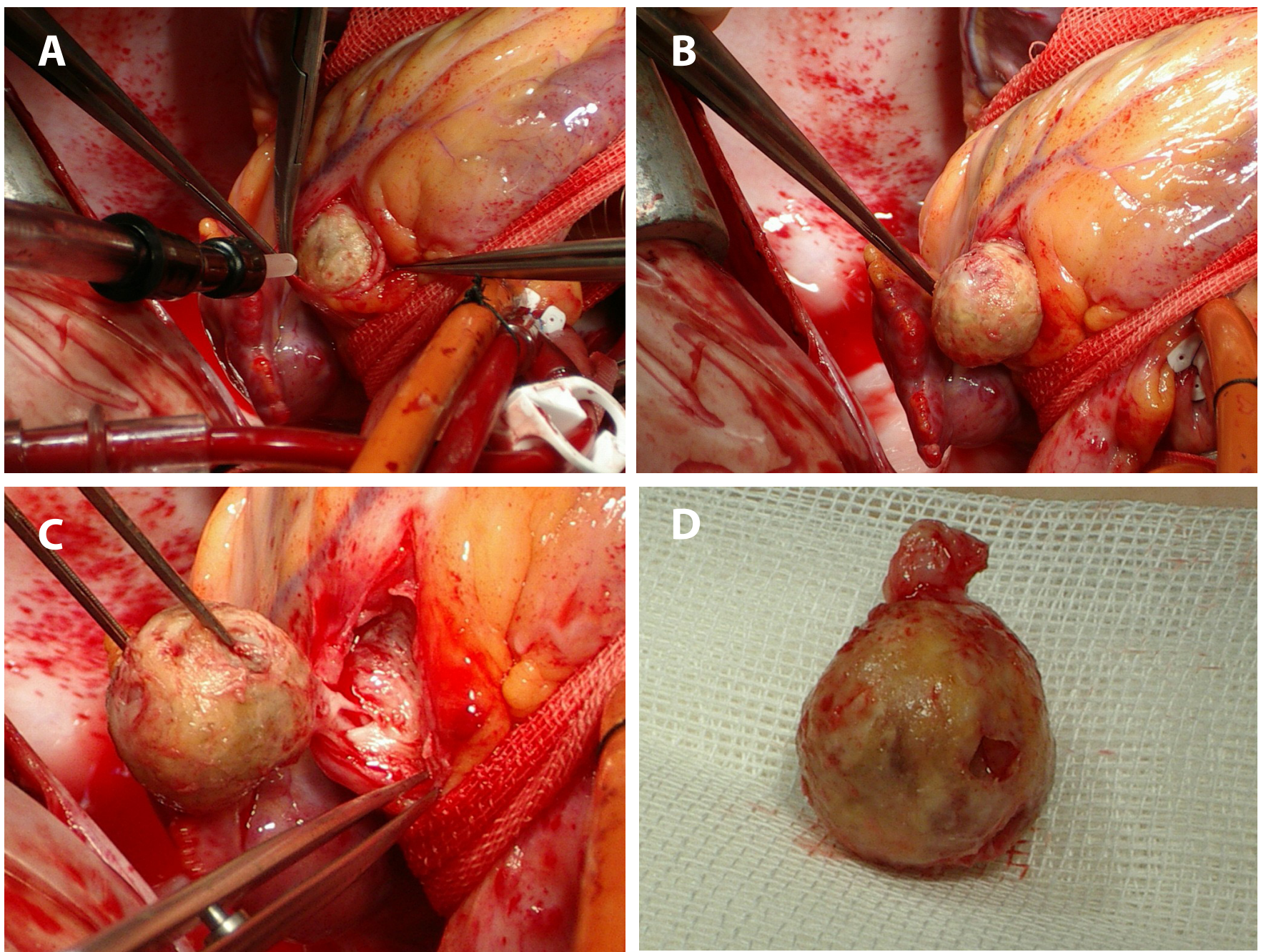

Fig. 3 - A: Perioperative view of the intracoronary hydatid cyst prior to enucleation (Size: $2 \times 2 \mathrm{~cm})$. B: Perioperative view of the intracoronary hydatid cyst, distally liberated but proximal attachment is intact. C: Perioperative view of the intracoronary hydatid cyst with proximal attachment (inferiorly located) intact. D: Enucleated intracoronary hydatid cyst, proximal attachment placed superiorly (size: $2 \times 2 \mathrm{~cm}$ ).

test is sensitive, the treatment strategy must not be directed by cyst serology. This is due to the fact that serology could be negative while hydatid cyst infestation is present or be positive for years while a treated and inactive cyst is present ${ }^{[10]}$. For this reason, considering all possibilities, oral albendazole treatment (10 mg/kg/day; 2x400 mg/day) postoperatively for 12 weeks was administered.

In young cases of hydatidosis, since clinical presentations resemble those of other conditions, without further studies it is hard to trace angina to cardiac involvement rather than pulmonary hydatidosis. Particularly in regions where hydatidosis is endemic, in differential diagnosis of young patients with coronary artery disease of unknown origin, cardiac hydatidosis must be considered. Moreover, while surgical intervention and medical therapy are planned for pulmonary hydatidosis, possibility of concomitant organ involvement must not be passed over.

\section{Authors' roles \& responsibilities}

UV Substantial contributions to the conception of the work: the analysis and interpretation of data for the work; final approval of the version to be published

AAA Substantial contributions to the conception of the work; the analysis and interpretation of data for the work; final approval of the version to be published

IK Substantial contributions to the conception of the work; the analysis and interpretation of data for the work; final approval of the version to be published

\section{REFERENCES}

1. Thameur H, Abdelmoula S, Chenik S, Bey M, Ziadi M, Mestiri T, et al. Cardiopericardial hydatid cyst. World J Surg. 2001;25(1):58-67.

2. Czermak BV, Akhan O, Hiemetzberger R, Zelger B, Vogel W, Jaschke W, 
et al. Echinococcosis of the liver. Abdom Imaging. 2008;33(2):133-43.

3. Kahlfuß S, Flieger RR, Roepke TK, Yilmaz K. Diagnosis and treatment of cardiac echinococcosis. Heart. 2016;102(17):1348-53.

4. Laglera S, Garcia-Enguita MA, Martinez-Gutierrez F, Ortega JP, GutierrezRodriguez A, Urieta A. A case of cardiac hydatidosis. Br J Anaesth. 1997;79(5):671-3.

5. Force L, Torres JM, Carrillo A, Busca J. Evaluation of eight serological tests in the diagnosis of human echinococcosis and follow-up. Clin Inf Dis. 1992;15(3):473-80.

6. Seth HS, Mishra P, Khandekar JV, Raut C, Mohapatra CKR, Ammannaya GKK. A concomitant intramyocardial and pulmonary hydatid cyst: a rare case report. Braz J Cardiovasc Surg. 2017;32(2):138-40.
7. Pakis I, Akyildiz EU, Karayel F, Turan AA, Senel B, Ozbay M, et al. Sudden death due to an unrecognized cardiac hydatid cyst: three medicolegal autopsy cases. J Forensic Sci. 2006;51(2):400-2.

8. Ben-Hamda K, Maatouk F, Ben-Farhat M, Betbout F, Gamra H, Addad $F$, et al. Eighteen-year experience with echinococcosis of the heart: clinical and echocardiographic features in 14 patients. Int J Cardiol. 2003;91(2-3):145-51.

9. Acikel S, Kiziltepe U, Turkvatan A, Cakici M, Koroglu DB, Sahpaz A, et al. Intracavitary cardiac hydatid cysts with a high risk of thromboemboli: invasive nature of cardiac echinococcosis. Herz. 2014;39(7):882-6.

10. NazligulY, Kucukazman M, Akbulut S. Role of chemotherapeutic agents in the management of cystic echinococcosis. Int Surg. 2015;100(1):112-4. 